\title{
Discrete time adaptive control for a MEMS gyroscope
}

\author{
Sungsu Park ${ }^{1, *, \dagger}$ and Roberto Horowitz ${ }^{2, \$}$ \\ ${ }^{1}$ Department of Aerospace Engineering, Sejong University, Seoul, Korea \\ ${ }^{2}$ Department of Mechanical Engineering, University of California, Berkeley, CA 94720, U.S.A.
}

\begin{abstract}
SUMMARY
This paper presents a discrete time version of the observer-based adaptive control system for micro-electromechanical systems gyroscopes, which can be implemented using digital processors. A stochastic analysis of this control algorithm is developed and it shows that the estimates of the angular rate and the fabrication imperfections are biased due to the signal discretization errors in the feedforward control path introduced by the sampler and holder. Thus, a two-rate discrete time control is proposed as a compromise between the measurement biases and the computational burden imposed on the controller. The convergence analysis of this algorithm is also conducted and an analysis method is developed for determining the trade-off between the controller sampling frequency and the magnitude of the angular rate estimate biased errors. All convergence and stochastic properties of a continuous time adaptive control are preserved, and this analysis is verified with computer simulations. Copyright (C) 2005 John Wiley \& Sons, Ltd.
\end{abstract}

KEY WORDS: MEMS; gyroscope; adaptive control; discrete time control; hybrid implementation

\section{INTRODUCTION}

Gyroscopes are commonly used sensors for measuring angular velocity in many areas of applications such as navigation, homing, and control stabilization. Although, conventional rotating wheel, fibre optic and ring laser gyroscopes have dominated a wide range of applications, they are too large and, most often too expensive to be used in most emerging applications. Recent advances in micro-machining technology have made the design and fabrication of micro-electro-mechanical systems (MEMS) gyroscopes possible. These devices are several orders of magnitude smaller than conventional mechanical gyroscopes, and can be fabricated in large quantities by batch processes. Thus, there is great potential to significantly reduce their fabrication cost. The emergence of MEMS gyroscopes is opening up new market opportunities and applications in the area of low-cost to medium performance inertial devices [1].

\footnotetext{
*Correspondence to: Sungsu Park, Department of Aerospace Engineering, Sejong University, 98 Gunja-dong, Kwangjin-gu, Seoul, Korea.

${ }^{\dagger}$ E-mail: sungsu@sejong.ac.kr

E-mail: horowitz@me.berkeley.edu
}

Contract/grant sponsor: DARPA; contract/grant number: N66001-97-C-8643

Copyright (C) 2005 John Wiley \& Sons, Ltd.

Received 15 October 2003 Accepted 14 January 2005 
Most MEMS gyroscopes are laminar vibratory mechanical structures fabricated on polysilicon or crystal silicon. Common fabrication steps include bulk micromachining, waferto-wafer bonding, surface micromachining, and high aspect ratio micromachining. Each of these fabrication steps involves multiple process steps such as deposition, etching and patterning of materials. Generally, every fabrication step contributes to imperfections in the gyroscope [2]. Fabrication imperfections that produce asymmetric structures, mis-alignment of actuation mechanism and deviations of the centre of mass from the geometric centre, result in undesirable, systematic perturbations in the form of mechanical and electrostatic forces, which degrade the performance of a gyroscope.

Currently, force-balancing feedback control schemes $[1,3,4]$ have been widely used to cancel the effect of off-diagonal terms in the stiffness matrix which is referred to as the quadrature error, and also to increase the bandwidth and dynamic range of the gyroscope beyond the openloop mode of operation. However, they rely on the exact measurement of input/output phase difference, and moreover are inherently sensitive to some types of fabrication imperfections which can be modelled as cross-damping terms, which produce zero-rate output (ZRO).

Recently, a new gyroscope operation mode and a corresponding adaptive control algorithm have been developed, which are well suited for the on-line compensation of imperfects and to operate in varying environments that affect the behaviour of a MEMS gyroscope [5]. This adaptive controlled gyroscope is self-calibrating, compensates for friction forces and fabrication imperfections that normally cause quadrature errors, and produces an unbiased angular velocity measurement that has no ZRO. The adaptive control algorithm presented in Reference [5] is a continuous time controller. It is assumed that the control and parameter adaptation laws are updated continuously in time. Although the implementation of such a controller is certainly possible utilizing analog circuits, it is of practical interest to explore the implementation of the adaptive control laws utilizing digital computers.

In this paper, we present a hybrid discrete/continuous time version of the observer-based adaptive control system developed in Reference [5], which can be implemented using digital processors. The control algorithm considered in this paper is not fully a discrete time controller, since only the feedback control, parameter adaptation algorithms and feedforward control law are implemented in discrete time, while the velocity observer is still implemented in continuous time.

In the next section, the dynamics of MEMS gyroscopes is presented, by accounting for the effect of fabrication imperfections. In Section 3, the observer-based adaptive control algorithm presented in Reference [5] is reviewed. The hybrid adaptive control law is developed in Section 4. In Section 5, the performance of the hybrid adaptive controlled gyroscope is analysed, including magnitude of parameter estimation biases, convergence rate and resolution estimation. Finally, computer simulations are performed in Section 6.

\section{DYNAMICS OF MEMS GYROSCOPES}

Common MEMS vibratory gyroscope configurations include a proof mass suspended by spring suspensions, and electrostatic actuations and sensing mechanisms for forcing an oscillatory motion and sensing the position and velocity of the proof mass. These mechanical components can be modelled as a mass, spring and damper system. Figure 1 shows a simplified model of a MEMS gyroscope having two degrees of freedom in the associated Cartesian reference frames. 


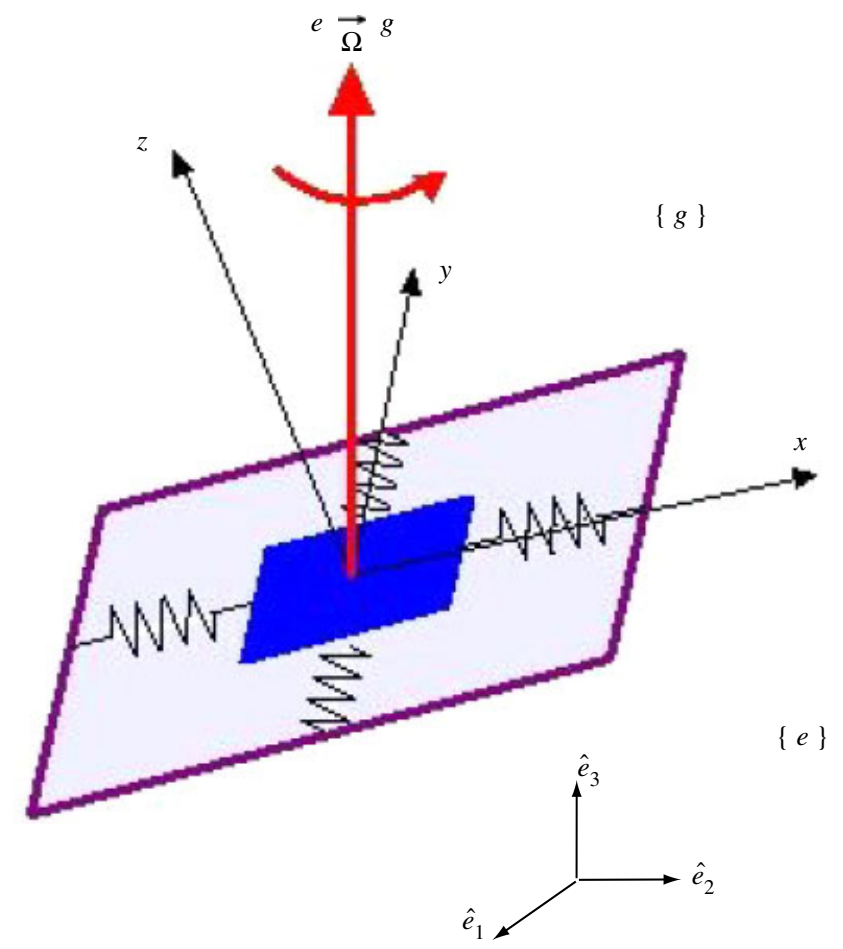

Figure 1. A model of a MEMS $z$-axis gyroscope.

Assuming that the motion of the proof mass is constrained to be only along the $x-y$ plane by making the spring stiffness in the $z$-direction much larger than in the $x$ - and $y$-directions, the measured angular rate is almost constant over a long enough time interval, and linear accelerations are cancelled out, either as an offset from the output response or by applying counter-control forces, then the equation of motion of a gyroscope is simplified as follows:

$$
\begin{aligned}
& m \ddot{x}+d_{1} \dot{x}+\left(k_{1}-m\left(\Omega_{y}^{2}+\Omega_{z}^{2}\right)\right) x+m \Omega_{x} \Omega_{y} y=\tau_{x}+2 m \Omega_{z} \dot{y} \\
& m \ddot{y}+d_{2} \dot{y}+\left(k_{2}-m\left(\Omega_{x}^{2}+\Omega_{z}^{2}\right)\right) y+m \Omega_{x} \Omega_{y} x=\tau_{y}-2 m \Omega_{z} \dot{x}
\end{aligned}
$$

where $x$ and $y$ are the co-ordinates of the proof mass relative to the gyro frame, $d_{1,2}, k_{1,2}$ are damping and spring coefficients, $\Omega_{x, y, z}$ are the angular velocity components along each axis of the gyro frame and $\tau_{x, y}$ are control forces. The two last terms in Equation (1), $2 m \Omega_{z} \dot{x}$ and $2 m \Omega_{z} \dot{y}$, are due to the Coriolis forces and are the terms which are used to measure the angular rate $\Omega_{z}$.

As seen in Equation (1), in an ideal gyroscope, only the component of the angular rate along the $z$-axis, $\Omega_{z}$, causes a dynamic coupling between the $x$ and $y$ axes, under the assumption that $\Omega_{x, y}^{2} \approx \Omega_{x} \Omega_{y} \approx 0$. In practice, however, small fabrication imperfections always occur, and also cause dynamic coupling between the $x$ and $y$ axes through the asymmetric spring and damping terms. Taking into account fabrication imperfections, the dynamic equations (1) are modified as 
follows [2]:

$$
\begin{aligned}
& m \ddot{x}+d_{x x} \dot{x}+d_{x y} \dot{y}+k_{x x} x+k_{x y} y=\tau_{x}+2 m \Omega_{z} \dot{y} \\
& m \ddot{y}+d_{x y} \dot{x}+d_{y y} \dot{y}+k_{x y} x+k_{y y} y=\tau_{y}-2 m \Omega_{z} \dot{x}
\end{aligned}
$$

Equation (2) is the governing equation for a MEMS $z$-axis gyroscope. Fabrication imperfections contribute mainly to the asymmetric spring and damping terms, $k_{x y}$ and $d_{x y}$. Therefore these terms are unknown, but can be assumed to be small. The $x$ and $y$ axes spring and damping terms are mostly known, but have small unknown variations from their nominal values. The proof mass can be determined very accurately. The components of angular rate along $x$ and $y$ axes are absorbed as part of the spring terms as unknown variations. Note that the spring coefficients $k_{x x}$ and $k_{y y}$ also include the electrostatic spring softness.

Based on $m, q_{0}$ and $\omega_{0}$, which are a reference mass, length and natural resonance frequency, respectively, where $m$ is a proof mass of the gyroscope, the non-dimensionalization of Equation (2) can be done as follows:

$$
\begin{aligned}
& \ddot{x}+d_{x x} \dot{x}+d_{x y} \dot{y}+\omega_{x}^{2} x+\omega_{x y} y=\tau_{x}+2 \Omega_{z} \dot{y} \\
& \ddot{y}+d_{x y} \dot{x}+d_{y y} \dot{y}+\omega_{x y} x+\omega_{y}^{2} y=\tau_{y}-2 \Omega_{z} \dot{x}
\end{aligned}
$$

where $\omega_{x}=\sqrt{k_{x x} /\left(m \omega_{0}^{2}\right)}, \omega_{y}=\sqrt{k_{y y} /\left(m \omega_{0}^{2}\right)}, \omega_{x y}=k_{x y} /\left(m \omega_{0}^{2}\right), d_{x x} \leftarrow d_{x x} /\left(m \omega_{0}\right), d_{y y} \leftarrow d_{y y} /$ $\left(m \omega_{0}\right), d_{x y} \leftarrow d_{x y} /\left(m \omega_{0}\right), \Omega_{z} \leftarrow \Omega_{z} / \omega_{0}, \tau_{x} \leftarrow \tau_{x} /\left(m \omega_{0}^{2} q_{0}\right)$ and $\tau_{y} \leftarrow \tau_{y} /\left(m \omega_{0}^{2} q_{0}\right)$.

\section{ADAPTIVE MODE OF OPERATION}

The aim of the adaptive mode of operation scheme presented in Reference [5] is to achieve (i) on-line compensation of fabrication imperfections, (ii) closed-loop identification of the angular rate, (iii) to attain a large bandwidth and dynamic range, and (iv) self-calibration operation. The adaptive mode of operation operates based on the observer-based adaptive control which needs only position measurements of the proof mass of the gyroscope. For convenience, governing Equation (3) of the MEMS gyroscopes is re-written as matrix form

$$
\ddot{q}+D \dot{q}+K q=\tau-2 \Omega \dot{q}+b
$$

where $b$ is Brownian noise and

$$
\begin{gathered}
q=\left[\begin{array}{l}
x \\
y
\end{array}\right], \quad \tau=\left[\begin{array}{l}
\tau_{x} \\
\tau_{y}
\end{array}\right], \quad \Omega=\left[\begin{array}{cc}
0 & -\Omega_{z} \\
\Omega_{z} & 0
\end{array}\right] \\
D=\left[\begin{array}{ll}
d_{x x} & d_{x y} \\
d_{x y} & d_{y y}
\end{array}\right], \quad K=\left[\begin{array}{cc}
\omega_{x}^{2} & \omega_{x y} \\
\omega_{x y} & \omega_{y}^{2}
\end{array}\right]
\end{gathered}
$$

The adaptive control problems of the gyroscope is formalized as follows: given Equation (4) with unknown constant parameters $D, K$ and $\Omega$, determine the control law $\tau$ based on measuring $q$, such that the dynamic range is constrained within a specified region and is estimated correctly. Note that direct measurement of the velocity of the proof mass is avoided in the problem formulation. This is because current velocity sensing circuitry technology produces a noise with 
spectral power that is 3-4 orders of magnitude larger than the ideally expected value, as compared with position sensing technology.

The observer-based adaptive control and parameter adaptation laws are introduced in Reference [5] as follows:

$$
\begin{gathered}
\tau=\tau_{0}+\hat{D} \dot{q}_{m}+\hat{R} q_{m}+2 \hat{\Omega} \dot{q}_{m} \\
\dot{\hat{R}}=\frac{1}{2} \gamma_{R}\left(\tau_{0} q_{m}^{\mathrm{T}}+q_{m} \tau_{0}^{\mathrm{T}}\right) \\
\dot{\hat{D}}=\frac{1}{2} \gamma_{D}\left(\tau_{0} \dot{q}_{m}^{\mathrm{T}}+\dot{q}_{m} \tau_{0}^{\mathrm{T}}\right) \\
\dot{\hat{\Omega}}=\gamma_{\Omega}\left(\tau_{0} \dot{q}_{m}^{\mathrm{T}}-\dot{q}_{m} \tau_{0}^{\mathrm{T}}\right) \\
\tau_{0}=-\gamma\left(\dot{\hat{q}}_{p}^{c}-\dot{q}_{m}\right)
\end{gathered}
$$

where $R=K-K_{m}, \hat{D}, \hat{R}$ and $\hat{\Omega}$ are estimates of $D, R$ and $\Omega$, and $\gamma=\operatorname{diag}\left\{\gamma_{1}, \gamma_{2}\right\}, \gamma, \gamma_{R}, \gamma_{D}$ and $\gamma_{\Omega}$ are adaptation gains. Reference trajectory, $q_{m}=\left[\begin{array}{ll}x_{m} & y_{m}\end{array}\right]^{\mathrm{T}}$, is updated by the following ideal oscillator:

$$
\ddot{q}_{m}+K_{m} q_{m}=0
$$

where $K_{m}=\operatorname{diag}\left\{\omega_{1}^{2}, \omega_{2}^{2}\right\}$ are the reference resonant modes of both axis. Note that the signal $q_{m}$ and $\dot{q}_{m}$ may be calculated and stored off-line, resulting in a significant reduction in the number of on-line computations.

The velocity estimate $\dot{\hat{q}}_{p}$ is corrupted by a measurement noise and produced by the following velocity observer:

$$
\begin{aligned}
& \dot{\hat{q}}_{p}^{c}=\hat{q}_{v}^{c}+L\left(q+n-\hat{q}_{p}^{c}\right) \\
& \dot{\hat{q}}_{v}^{c}=-K_{m} \hat{q}_{p}^{c}
\end{aligned}
$$

where $\hat{q}_{p}^{c}$ is the estimate of the position, $\hat{q}_{v}^{c}$ is an additional state of the velocity observer, $L$ is a observer gain matrix given by $L=\operatorname{diag}\left\{L_{1}, L_{2}\right\}$ and $n$ is a position measurement noise, which is assumed to be uncorrelated with Brownian noise $b$. The estimated power spectral densities of the position measurement noise $\left(S_{\mathrm{p}}\right)$ and Brownian noise $\left(S_{\mathrm{b}}\right)$ are given by [6]

$$
S_{\mathrm{p}}=\left(\frac{2 C_{0}+C_{\mathrm{p}}}{2 V_{0}(\mathrm{~d} C / \mathrm{d} y)}\right)^{2} 4 k_{\mathrm{B}} T R_{\mathrm{w}}, \quad S_{\mathrm{b}}=\frac{4 k_{\mathrm{B}} T d}{m^{2}}
$$

where $k_{\mathrm{B}}, C_{\mathrm{p}}, C_{0}, R_{\mathrm{w}}, T, d$ and $m$ are, respectively, Boltzmann's constant, the device's parasite capacitance, nominal sensing capacitance, wiring resistance, absolute temperature, damping coefficient and mass. Both are assumed zero-mean white noises.

We present the following two theorems without proof. The proofs can be found in Reference [5].

\section{Theorem 1 (Stability)}

Given the observer (8), the adaptive control (5) and parameter adaptation laws (6), it is always possible to choose a velocity observer gain $L$, which makes the trajectory error, $e_{\mathrm{p}}=q-q_{m}$, 
trajectory estimation errors, $\tilde{q}_{p}=\hat{q}_{p}-q$ and $\tilde{q}_{v}=\hat{q}_{v}-\dot{q}$, and their time derivatives converge locally, uniformly and exponentially to zero.

Theorem 2 (Persistent excitation condition)

With control law (5), parameter adaptation laws (6), and the observer (8), if the gyroscope is controlled to follow the mode-unmatched reference model, i.e. $\omega_{1} \neq \omega_{2}$, the persistent excitation condition is satisfied and all unknown gyroscope parameters, including the angular rate, are estimated correctly.

Theorems 1 and 2 show that the motion of a mode-unmatched gyroscope, in which the resonance frequency of the $x$-axis is different from that of the $y$-axis, has sufficient persistence of excitation to permit the identification of all major fabrication imperfections as well as 'input' angular rate. This means that adaptive controlled gyroscope has no ZRO and is self-calibrating. The main advantages of the adaptive mode of operation include self-calibration, large robustness to parameter variations, and no zero-rate output. Moreover, the adaptive controller design is also easy to implement in high $Q$ systems. Thus, the noise properties associated with a high $Q$ system can be fully utilized. Another advantage of the adaptive mode of operation is that it is easy to adjust the trade-off between bandwidth and resolution by simply adjusting the angular rate adaptation gain.

\section{HYBRID ADAPTIVE CONTROL LAW}

\subsection{Discrete time adaptive control}

We now consider the implementation of the adaptive control algorithm described by Equations (5)-(6) utilizing a digital computer. The control law and parameter adaptation algorithm will be implemented in discrete time, while the observer (8) will be implemented in continuous time. Thus, the adaptive algorithm can no longer be analysed as a continuous time system, but rather as a hybrid system which includes both discrete time and continuous time algorithms. For convenience, let us define an adaptation gain matrix $\Gamma$, a signal regressor $W\left(q_{m}, \dot{q}_{m}\right)$, and an unknown parameter vector $\theta$ as follows:

$$
\begin{gathered}
\Gamma=\operatorname{diag}\left\{\gamma_{R}, \frac{1}{2} \gamma_{R}, \gamma_{R}, \gamma_{D}, \frac{1}{2} \gamma_{D}, \gamma_{D}, \frac{1}{2} \gamma_{\Omega}\right\} \\
W^{\mathrm{T}}\left(q_{m}, \dot{q}_{m}\right)=\left[\begin{array}{ccccccc}
x_{m} & y_{m} & 0 & \dot{x}_{m} & \dot{y}_{m} & 0 & -2 \dot{y}_{m} \\
0 & x_{m} & y_{m} & 0 & \dot{x}_{m} & \dot{y}_{m} & 2 \dot{x}_{m}
\end{array}\right] \\
\theta^{\mathrm{T}}=\left[\begin{array}{lllllll}
r_{x x} & r_{x y} & r_{y y} & d_{x x} & d_{x y} & d_{y y} & \Omega_{z}
\end{array}\right]
\end{gathered}
$$

where $r_{i j}, d_{i j}$ and $\Omega_{z}$ are, respectively, elements of $R, D$ and $\Omega$. Also, let us define the sampling index $k$ and the sampling time $\Delta t$, such that $t_{k}=k \Delta t$. We define the hold operator $H$ such that, $H[s(k)]$ denotes

$$
H[s(k)]=s\left(t_{k}\right) \text { for } t_{k} \leqslant t<t_{k+1}
$$

Using $W_{m}(t)$ as a short hand notation for $W\left(q_{m}, \dot{q}_{m}\right)$, let us define $W_{m}(k)$ as

$$
W_{m}(k)=W_{m}\left(t_{k}\right)=W\left(q_{m}\left(t_{k}\right), \dot{q}_{m}\left(t_{k}\right)\right)
$$


Now, define the discrete time adaptive control law as

$$
\tau(t)=-\gamma H\left[\left(\dot{\hat{q}}_{p}^{c}(k)-\dot{q}_{m}(k)\right)+\delta q(k)\right]+H\left[W_{m}^{\mathrm{T}}(k) \hat{\theta}(k)\right]
$$

where $\delta q(k)$ is a quantization noise of the velocity estimate. Because of finite word length error or digital computational error, the actual signal $W_{m}(k)$ must also be modelled as $W_{a}(k)=$ $W_{m}(k)+\delta W_{m}(k)$, where $\delta W_{m}(k)$ is a finite word length error. However, since the signal $W_{m}(k)$ is known in advance and can be generated by the computer, its finite word length error can be made arbitrary small and will be neglected here. Note that the signal $W_{m}(k)$ may be calculated and stored in an off-line fashion, resulting in a significant reduction in the number of on-line computations.

The parameter estimate sequence in Equation (10) is updated by the following approximated continuous time parameter adaptation law:

$$
\int_{t_{k}}^{t_{k+1}} \dot{\hat{\theta}}(t) \mathrm{d} t=\int_{t_{k}}^{t_{k+1}}-\Gamma H\left[W_{m}(k) \gamma\left(\dot{\hat{q}}_{p}^{c}(k)-\dot{q}_{m}(k)+\delta q(k)\right)\right] \mathrm{d} t
$$

Thus,

$$
\hat{\theta}(k+1)=\hat{\theta}(k)-\Gamma W_{m}(k) \gamma\left(\dot{\hat{q}}_{p}^{c}(k)-\dot{q}_{m}(k)+\delta q(k)\right) \Delta t
$$

while the observer signals $\dot{\hat{q}}_{p}^{c}(t)$ and $\hat{q}_{v}^{c}(t)$ are still updated by the continuous time observer (8) and sampled at every $\Delta t$.

\subsection{Stability analysis}

In order to derive the closed loop error equations, we apply the control law (10) and the adaptation algorithm (12) to the system equation (4) and observer (8). Define the signal discretization error function as

$$
\Delta W_{m}(t)=H\left[W_{m}(k)\right]-W_{m}(t)
$$

then the trajectory error equations are

$$
\begin{aligned}
\ddot{e}_{p}+(D+2 \Omega) \dot{e}_{p}+K_{m} e_{p}= & -\gamma H\left[\dot{e}_{p}(k)+\dot{\tilde{q}}_{p}(k)+\delta_{q}(k)\right] \\
& +H\left[W_{m}^{\mathrm{T}}(k) \hat{\theta}(k)\right]-W_{m}^{\mathrm{T}}(t) \theta-R e_{p}+b
\end{aligned}
$$

where

$$
\begin{aligned}
H\left[W_{m}^{\mathrm{T}}(k) \hat{\theta}(k)\right]-W_{m}^{\mathrm{T}}(t) \theta & =H\left[W_{m}^{\mathrm{T}}(k) \tilde{\theta}(k)\right]+\left(H\left[W_{m}^{\mathrm{T}}(k)\right]-W_{m}^{\mathrm{T}}(t)\right) \theta \\
& =H\left[W_{m}^{\mathrm{T}}(k) \tilde{\theta}(k)\right]+\Delta W_{m}^{\mathrm{T}}(t) \theta
\end{aligned}
$$

where $\tilde{\theta}=\hat{\theta}-\theta$. The trajectory estimation error equations are

$$
\begin{aligned}
\dot{\tilde{q}}_{p}= & \tilde{q}_{v}-L \tilde{q}_{p}+L n \\
\dot{\tilde{q}}_{v}= & -K_{m} \tilde{q}_{p}+(D+2 \Omega) \dot{e}_{p} \\
& +\gamma H\left[\dot{e}_{p}(k)+\dot{\tilde{q}}_{p}(k)+\delta q(k)\right] \\
& -H\left[W_{m}^{\mathrm{T}}(k) \tilde{\theta}(k)\right]-\Delta W_{m}^{\mathrm{T}}(t) \theta+R e_{p}-b
\end{aligned}
$$


where $e_{p}=q-q_{m}, \tilde{q}_{p}=\hat{q}_{p}^{c}-q$, and $\tilde{q}_{v}=\hat{q}_{v}^{c}-\dot{q}$. Equations (14) and (15) can be described compactly by the sum of a linear known and a linear unknown term as follows:

$$
\begin{aligned}
\dot{x}_{c}= & A_{c} x_{c}+A_{c u} x_{c} \\
& -B_{c} \gamma H\left[\dot{e}_{p}(k)+\tilde{q}_{v}(k)-L \tilde{q}_{p}(k)+L n_{s}(k)+\delta q(k)\right] \\
& +B_{c} H\left[W_{m}^{\mathrm{T}}(k) \tilde{\theta}(k)\right]+B_{c} \Delta W_{m}^{\mathrm{T}}(t) \theta+B_{c} b+C_{c} n
\end{aligned}
$$

where $n_{s}(k)$ is the sampled measurement noise, where $n_{s}(k) \sim\left(0, S_{p} / \Delta t\right), x_{c}=\left[\begin{array}{llll}e_{p} & \dot{e}_{p} & \tilde{q}_{p} & \tilde{q}_{v}\end{array}\right]^{\mathrm{T}}$ and

$$
A_{c}=\left[\begin{array}{cccc}
0 & I & 0 & 0 \\
-K_{m} & 0 & 0 & 0 \\
0 & 0 & -L & I \\
0 & 0 & -K_{m} & 0
\end{array}\right], \quad A_{c u}=\left[\begin{array}{cccc}
0 & 0 & 0 & 0 \\
-R & -(D+2 \Omega) & 0 & 0 \\
0 & 0 & 0 & 0 \\
R & (D+2 \Omega) & 0 & 0
\end{array}\right], \quad B_{c}=\left[\begin{array}{c}
0 \\
I \\
0 \\
-I
\end{array}\right], \quad C_{c}=\left[\begin{array}{l}
0 \\
0 \\
L \\
0
\end{array}\right]
$$

Since the 'control input' in Equation (16)

$$
\begin{aligned}
& -\gamma H\left[\dot{e}_{p}(k)+\tilde{q}_{v}(k)-L \tilde{q}_{p}(k)+L n_{s}(k)+\delta q(k)\right] \\
& \quad+H\left[W_{m}^{\mathrm{T}}(k) \tilde{\theta}(k)\right]
\end{aligned}
$$

is held constant over the sampling period and the parameters are updated in a discrete time, it is convenient for the further analysis to discretize the trajectory and trajectory estimate error systems (16) as follows:

$$
\begin{aligned}
x_{c}(k+1)= & \left(I+A_{c} \Delta t\right) x_{c}(k)+A_{c u} \Delta t x_{c}(k)+O_{1}\left(\Delta t^{2}\right) x_{c}(k) \\
& -B_{c} \Delta t \gamma\left[\dot{e}_{p}(k)+\tilde{q}_{v}(k)-L \tilde{q}_{p}(k)+L n_{s}(k)+\delta q(k)\right] \\
& +B_{c} \Delta t W_{m}^{\mathrm{T}}(k) \tilde{\theta}(k)+O_{2}\left(\Delta t^{2}\right)+C(k) \theta+B_{c} b_{d}(k)+C_{c} n_{d}(k)
\end{aligned}
$$

where $O_{1,2}\left(\Delta t^{2}\right)$ are the terms of order $\Delta t^{2}$ and denote how the remainder (the error) depends on $\Delta t$, and

$$
C(k)=\int_{t_{k}}^{t_{k+1}} e^{\left(A_{c}+A_{c u}\right)\left(t_{k+1}-t\right)} B_{c} \Delta W_{m}^{\mathrm{T}}(t) \mathrm{d} t
$$

where $b_{d}(k) \sim\left(0, S_{\mathrm{b}} \Delta t\right), n_{d} \sim\left(0, S_{\mathrm{p}} \Delta t\right)$. Notice that, in the numerical integration of Equation (16), the intensity of the sampled noises $b_{d}(k)$ and $n_{d}(k)$ can be approximately calculated by multiplying the intensity of noises $b(t)$ and $n(t)$ by $\Delta t$. 
Utilizing the parameter adaptation law

$$
\begin{aligned}
\tilde{\theta}(k+1)= & \tilde{\theta}(k)-\Delta t \Gamma W_{m}(k) \gamma\left(\dot{e}_{p}(k)+\dot{\tilde{q}}_{p}(k)+\delta q(k)\right) \\
= & \tilde{\theta}(k)-\Delta t \Gamma W_{m}(k) \gamma\left(\dot{e}_{p}(k)+\tilde{q}_{v}(k)\right. \\
& \left.-L \tilde{q}_{p}(k)+L n_{s}(k)+\delta q(k)\right)
\end{aligned}
$$

the extended error dynamics can be described by the following discrete time system:

$$
\begin{aligned}
x_{d}(k+1)= & A_{d}(k) x_{d}(k)+A_{d u} x_{d}(k)+O\left(\Delta t^{2}\right) x_{d}(k) \\
& +C_{d}(k) \theta+G_{d}(k) w_{d}(k)
\end{aligned}
$$

where $x_{d}=\left[\begin{array}{lllll}e_{p} & \dot{e}_{p} & \tilde{q}_{p} & \tilde{q}_{v} & \tilde{\theta}\end{array}\right]^{\mathrm{T}}, w_{d}=\left[\begin{array}{lll}b_{d} & n_{d} & \delta q_{d}\end{array}\right]^{\mathrm{T}}$ and

$$
\begin{aligned}
& A_{d}(k)=\left[\begin{array}{ccccc}
I & \Delta t & 0 & 0 & 0 \\
-K_{m} \Delta t & I-\gamma \Delta t & \gamma L \Delta t & -\gamma \Delta t & W_{m}^{\mathrm{T}} \Delta t \\
0 & 0 & I-L \Delta t & \Delta t & 0 \\
0 & \gamma \Delta t & -\left(K_{m}+\gamma L\right) \Delta t & I+\gamma \Delta t & -W_{m}^{\mathrm{T}} \Delta t \\
0 & -\Gamma W_{m} \gamma \Delta t & \Gamma W_{m} \gamma L \Delta t & -\Gamma W_{m} \gamma \Delta t & I
\end{array}\right] \\
& A_{d u}=\left[\begin{array}{ccccc}
0 & 0 & 0 & 0 & 0 \\
-R \Delta t & -(D+2 \Omega) \Delta t & 0 & 0 & 0 \\
0 & 0 & 0 & 0 & 0 \\
R \Delta t & (D+2 \Omega) \Delta t & 0 & 0 & 0 \\
0 & 0 & 0 & 0 & 0
\end{array}\right] \\
& G_{d}(k)=\left[\begin{array}{ccc}
0 & 0 & 0 \\
I & -\gamma L & -\gamma \\
0 & L & 0 \\
-I & \gamma L & \gamma \\
0 & -\Gamma W_{m}(k) \gamma L & -\Gamma W_{m}(k) \gamma
\end{array}\right] \\
& C_{d}(k)=\left[\begin{array}{c}
C(k) \\
0
\end{array}\right], \quad w_{d}(k) \sim\left(0, S_{d}\right)
\end{aligned}
$$


where $S_{d}=\operatorname{diag}\left(S_{c} \Delta t, S_{\text {quan }} \Delta t^{2}\right), S_{c}=\operatorname{diag}\left\{S_{\mathrm{b}}, S_{\mathrm{p}}\right\}$ and $S_{\text {quan }}$ is the power spectral density of the quantization noise for $\delta q$.

The higher order term, $O\left(\Delta t^{2}\right)$, is composed of $O_{1}\left(\Delta t^{2}\right)$ and $O_{2}\left(\Delta t^{2}\right)$, and is given by

$$
\begin{gathered}
O\left(\Delta t^{2}\right)=\left[\begin{array}{cc}
o_{1} & o_{2} \\
0 & 0
\end{array}\right] \Delta t^{2}+\cdots \Delta t^{3}+\cdots \\
o_{1}=\frac{1}{2}\left(A_{c}+A_{c u}\right)\left(\left(A_{c}+A_{c u}\right)+B_{c}[0-\gamma \gamma L-\gamma]\right) \\
o_{2}=\frac{1}{2}\left(A_{c}+A_{c u}\right) B_{c} W_{m}^{\mathrm{T}}(k)
\end{gathered}
$$

In order to prove the stability of the closed-loop system, we consider the stochastic expectation propagation of Equation (19). Since the propagation equation has the same form as its deterministic counterpart, we can consider the deterministic case, i.e. $w_{d}(k)=0$. Since $A_{d}(k)$ in Equation (19) is a periodic discrete time-varying matrix with period $k_{\mathrm{T}}=4 \pi^{2} /\left(\omega_{1} \omega_{2} \Delta t\right)$, where $\omega_{1}$ and $\omega_{2}$ are the model reference frequencies, we can utilize a similar analytical procedure to be the one that was employed in Reference [5] to analyse the stability of the continuous time varying error dynamics. To do that, we consider a discrete time version of Floquet theory [5, 7].

\section{Lemma (Discrete time Floquet theory)}

Consider the following discrete time periodic time-varying linear system with period $k_{\mathrm{T}}$

$$
x(k+1)=A(k) x(k), \quad \text { where } A(k)=A\left(k+k_{\mathrm{T}}\right)
$$

where $A(k)$ is a non-singular matrix. Then, there exists a periodic transformation which converts the periodic time-varying linear system to a time invariant linear system.

Now, we are ready to prove the stability of the error dynamics of Equation (19).

\section{Theorem 3 (Stability)}

Consider the adaptive control law and adaptation laws, given by Equations (10) and (12), and the adaptive observer (8), which result in the error dynamics (19). If the sampling time $\Delta t$ is sufficiently small, it is always possible to choose an observer gain $L$ which will result in all error signals, $e_{p}, \dot{e}_{p}, \quad \tilde{q}_{p}, \tilde{q}_{v}$ and $\tilde{\theta}$ being bounded. These bounds are a function of the discretization error introduced by sampling and the zero-order hold.

Proof

Let $\Phi(k)$ be a state transition matrix associated with the periodic matrix $A_{d}(k)$ in Equation (19), i.e.

$$
\Phi_{d}(k+1)=A_{d}(k) \Phi_{d}(k)
$$

then the state transition matrix can be written as a product of two matrices as

$$
\Phi_{d}(k)=F_{d}(k) \bar{A}_{d}^{k}
$$

where $F_{d}(k)$ is a discrete periodic nonsingular matrix with period $k_{\mathrm{T}}$ which satisfies condition, $F_{d}(0)=F_{d}\left(k_{\mathrm{T}}\right)=I . \bar{A}_{d}$ is a constant matrix and the stability of the linear time varying known dynamics

$$
x_{d n}(k+1)=A_{d}(k) x_{d n}(k)
$$


is determined by eigenvalues of $\bar{A}_{d}$. Similarly to the continuous time case, an appropriate observer gain $L$ always exists such that the matrix $\bar{A}_{d}$ will be stable. In order to determine $F_{d}(k)$, the state transition matrix $\Phi_{d}(k)$ must be computed. Unfortunately, it is hard to analytically compute $\Phi_{d}(k)$. Instead, the transition matrix at the end of one period is numerically computed from Equation (21) utilizing the initial condition $\Phi_{d}(0)=I$ and $\bar{A}_{d}$ is obtained from $\bar{A}_{d}=$ $\Phi^{-k_{\mathrm{T}}}\left(k_{\mathrm{T}}\right)$.

The remainder of the stability proof is based on the Lyapunov function approach. Consider the following Lyapunov function candidate:

$$
V(k)=x_{d}^{\mathrm{T}}(k) F_{d}^{-\mathrm{T}}(k) M F_{d}^{-1}(k) x_{d}(k)
$$

where $M$ is the solution of Lyapunov function, $\bar{A}_{d}^{\mathrm{T}} M \bar{A}_{d}-M=-I$. Since $\bar{A}_{d}$ is asymptotically stable, $M>0$ and $F_{d}^{-\mathrm{T}}(k) M F_{d}^{-1}(k)>0$ for all $k \geqslant 0$. Using the following relationships, $\bar{A}_{d}=F_{d}^{-1}(k+1) A_{d}(k) F_{d}(k)$, and calculating the difference of $V$ along the trajectory (19) gives

$$
\begin{aligned}
\Delta V(k)= & V(k+1)-V(k) \\
\leqslant & -\alpha_{\min }^{2}\left\|x_{d}\right\|^{2}+\alpha_{\max }^{2} \beta^{2} \lambda_{\max }(M)\left\|x_{d}\right\|^{2} \\
& +2 \alpha_{\max }^{2} \varepsilon \beta \lambda_{\max }(M)\left\|x_{d}\right\|^{2} \\
& +\alpha_{\max }^{2} \eta^{2} \lambda_{\max }(M)\|\theta\|^{2} \\
& +2 \alpha_{\max }^{2} \eta \varepsilon \lambda_{\max }(M)\left\|x_{d}\right\|\|\theta\| \\
& +2 \alpha_{\max }^{2} \beta \eta \lambda_{\max }(M)\left\|x_{d}\right\|\|\theta\|
\end{aligned}
$$

where $\quad \alpha_{\min }=\min _{0 \leqslant k \leqslant k_{T}}\left\|F^{-1}(k)\right\|, \quad \alpha_{\max }=\max _{0 \leqslant k \leqslant k_{T}}\left\|F^{-1}(k)\right\|, \quad \eta=\max _{0 \leqslant k \leqslant k_{T}}\left\|C_{d}(k)\right\|$, $\beta=\left\|A_{d u}+O\left(\Delta t^{2}\right)\right\|, \varepsilon=\left\|\bar{A}_{d}\right\|$, and $\lambda_{\max }(M)$ is a maximum eigenvalue of $M$. If the signal discretization error $\Delta W_{m}(t)=H\left[W_{m}(k)\right]-W_{m}(t)$ is zero, or equally $\eta=0$, the origin of the state space $\left(e_{p}, \dot{e}_{p}, \tilde{q}_{p}, \tilde{q}_{v}, \tilde{\theta}\right)$ is guaranteed to be locally uniformly exponentially stable within the domain of $\alpha_{\min }^{2}>\alpha_{\max }^{2} \lambda_{\max }(M) \beta(\beta+2 \varepsilon)$. Since some amount of discretization error is always present, the trajectory, trajectory estimation and parameter estimation errors will not converge to zero, but rather to a compact residual set. This residual set is defined by

$$
\aleph=\left\{x_{d}:\left\|x_{d}\right\| \leqslant \eta\|\theta\|\left(\frac{s_{2}+\sqrt{s_{2}^{2}+s_{1} s_{3}}}{s_{1}}\right)\right\}
$$

where

$$
\begin{aligned}
& s_{1}=\alpha_{\min }^{2}-\alpha_{\max }^{2} \lambda_{\max }(M) \beta(\beta+2 \varepsilon) \\
& s_{2}=\alpha_{\max }^{2} \lambda_{\max }(M)(\beta+\varepsilon), \quad s_{3}=\alpha_{\max }^{2} \lambda_{\max }(M)
\end{aligned}
$$

and the theorem is proved.

The bound of $\Delta t$ for the analysis of Theorem 3 to be valid may be computed from the condition of $s_{1}>0$, i.e.

$$
\Delta t<\frac{\sqrt{\left\|A_{c u}\right\|^{2}+4\|\tilde{O}(\cdot)\| \beta^{*}}-\left\|A_{c u}\right\|}{2\|\tilde{O}(\cdot)\|} \approx \frac{\beta^{*}}{\left\|A_{c u}\right\|}
$$


where

$$
\beta^{*} \approx \frac{\alpha_{\min }^{2}}{2 \varepsilon \alpha_{\max }^{2} \lambda_{\max }(M)}, \quad O\left(\Delta t^{2}\right)=\Delta t^{2} \tilde{O}(\cdot)
$$

\section{PERFORMANCE ANALYSIS}

\subsection{Magnitude of parameter estimation biases}

As discussed in the stability analysis, the introduction of the signal discretization error function $\Delta W_{m}(t)$, which was defined in Equation (13), prevents the error dynamics from asymptotically converging to zero and introduces bias in the estimates of the angular rate and fabrication imperfections. In order to reduce bias of the estimates, it is necessary to make this discretization error small by achieving a fast computation rate in the feedforward control path. To choose an appropriate computation rate in the feedforward control path, it is necessary to determine the relationship between the computation rate and the magnitude of the bias estimate.

Consider the signal discretization error term $C(k) \theta$ in Equation (17) and its average power. If its power is equal to the power spectral density of a fictitious noise term $G_{c} w(k)$, where

$$
G_{c}=\left[\begin{array}{cc}
0 & 0 \\
I & -\gamma L \\
0 & L \\
-I & \gamma L
\end{array}\right], \quad w=\left[\begin{array}{l}
b_{d} \\
n_{d}
\end{array}\right] \sim\left(0, S_{c} \Delta t\right)
$$

where $S_{c}=\operatorname{diag}\left\{S_{\mathrm{b}}, S_{\mathrm{p}}\right\}$, the effect of the signal discretization error on the error dynamics of $e_{p}, \dot{e}_{p}, \tilde{q}_{p}, \tilde{q}_{v}$ and $\tilde{\theta}$ will be same to that of the noise in the stochastic average sense. Thus,

$$
A V G\left(C(k) \theta \theta^{\mathrm{T}} C^{\mathrm{T}}(k)\right)=G_{c} S_{c} G_{c}^{\mathrm{T}} \Delta t
$$

Equation (23) can be used to determine the following bound:

$$
\max \|C(k) \theta\|^{2}=\left\|G_{c}\right\|^{2}\left\|S_{c}\right\| \Delta t
$$

Using Euler's numerical integration approximation, $\|C(k) \theta\|$ can be further approximated as follows:

$$
\|C(k) \theta\| \leqslant\left\|B_{c}\right\|\left\|\Delta W_{m}^{\mathrm{T}}(t) \theta\right\|_{\max } \Delta t
$$

The right-hand side of Equation (25) can be further expanded as follows:

$$
\begin{aligned}
\left\|\Delta W_{m}^{\mathrm{T}}(t) \theta\right\| & =\left\|(D+2 \Omega) \Delta \dot{q}_{m}(t)+R \Delta q_{m}(t)\right\| \\
& \leqslant\left\|\Delta \dot{q}_{m}(t)\right\|\|D+2 \Omega\|+\left\|\Delta q_{m}(t)\right\| R \| \\
& \leqslant \Delta t\left\{\sqrt{X_{0}^{2} \omega_{1}^{4}+Y_{0}^{2} \omega_{2}^{4}}\|D+2 \Omega\|+\sqrt{X_{0}^{2} \omega_{1}^{2}+Y_{0}^{2} \omega_{2}^{2}}\|R\|\right\}
\end{aligned}
$$


Under the decoupling condition of the angular estimate and fabrication imperfection estimates [5], i.e. $X_{0} \omega_{1}=Y_{0} \omega_{2}$, Equation (24) becomes

$$
2 \Delta t^{2} X_{0} \omega_{1}\left(\omega_{1}\|D+2 \Omega\|+\|R\|\right)=\left\|G_{c}\right\| \sqrt{\Delta t\left\|S_{c}\right\|}
$$

Therefore, the required sampling time to achieve parameter estimation bias magnitude below or equal to the Brownian and position measurement noise floor is given by

$$
\Delta t=\left(\frac{\left\|G_{c}\right\| \sqrt{\left\|S_{c}\right\|}}{2 X_{0} \omega_{1}\left(\omega_{1}\|D+2 \Omega\|+\|R\|\right)}\right)^{2 / 3}
$$

where $D$ is the damping matrix, $R$ is the resonant frequency modelling error matrix, and $\Omega$ is the unknown 'input' angular rate. Equation (27) is a useful criterion for choosing an approximate computation rate for the feedforward control path. Equation (27) suggests that the magnitude of biases of the angular rate and fabrication imperfection estimates is proportional to the computation rate elevated to the $\frac{3}{2}$ as shown in Figure 2. However, it is important to note that 'magnitude' in this context means the norm of a vector composed of the angular rate and fabrication imperfection estimate errors. Thus, individual biases such as the angular rate estimation bias, may be equal to or less than this estimate.

When the sampling rate of the discrete time control algorithm in Equations (10) and (12) is increased significantly, the computational burden of the control algorithm may become too high, and may cause unnecessarily over-sampling in the parameter adaptation algorithm. This analysis suggests the introduction of a two-rate discrete time control as a compromise between

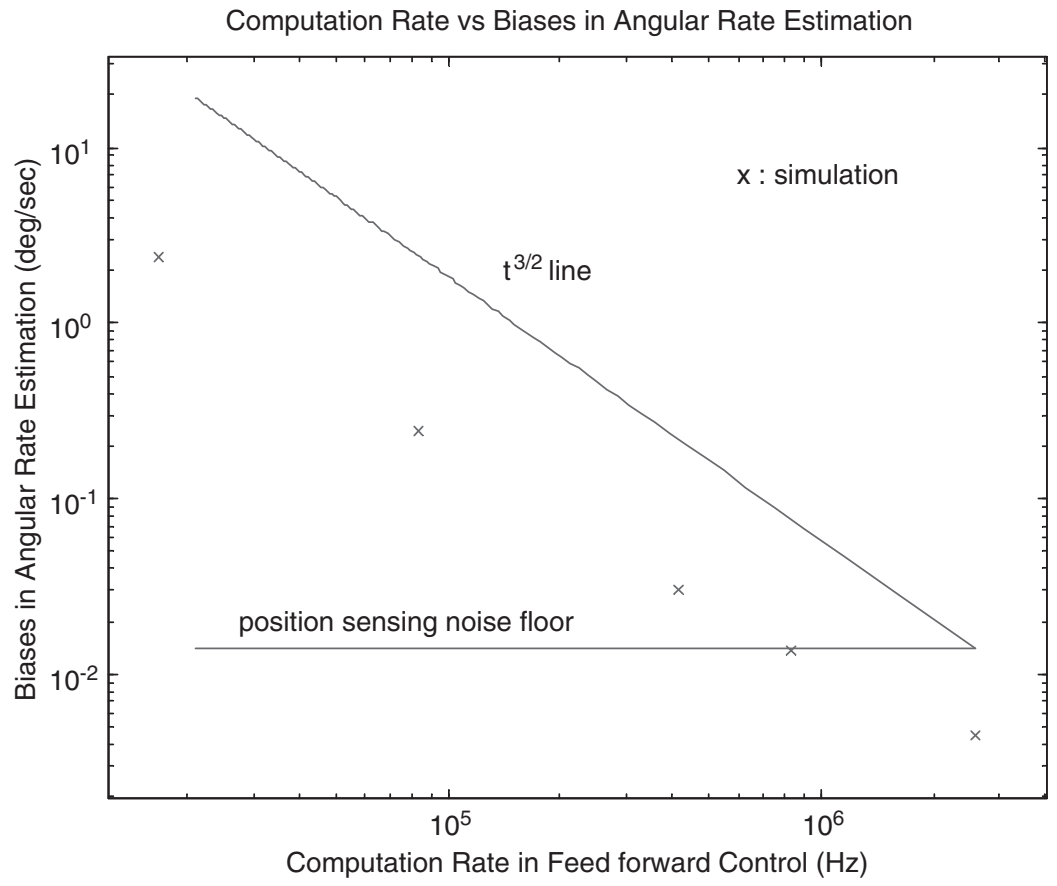

Figure 2. Computation rate in the feed forward control path vs bias in the parameter estimations. 
minimizing bias estimate due to discretization errors and attaining an implemental controller computational burden. In this two-rate discrete time controller, the parameter adaptation algorithm is updated at a slower rate than the reference signal which is used in the feedforward control.

\subsection{Convergent rate analysis}

In order to apply averaging analysis, consider the error dynamics given by Equations (16) and (18). We will ignore the signal discretization error in the feedforward control path which causes the bias of the states. As in the continuous time counter part, we first assume that all noise terms are zero. If we do a state co-ordinate transformation from $x_{c}=\left[\begin{array}{llll}e_{p} & \dot{e}_{p} & \tilde{q}_{p} & \tilde{q}_{v}\end{array}\right]^{\mathrm{T}}$ to $x_{a}=\left[\begin{array}{llll}e_{p} & \dot{e}_{p} & \tilde{q}_{p} & \dot{\tilde{q}}_{p}\end{array}\right]^{\mathrm{T}}$, then

$$
\begin{aligned}
& \dot{x}_{a}= A_{a} x_{a}+A_{c u} x_{a} \\
&-B_{c} \gamma H\left[\dot{e}_{p}(k)+\dot{\tilde{q}}_{p}(k)\right]+B_{c} H\left[W_{m}^{\mathrm{T}}(k) \tilde{\theta}(k)\right] \\
& \tilde{\theta}(k+1)=\tilde{\theta}(k)-\Delta t \Gamma W_{m}(k) \gamma\left(\dot{e}_{p}(k)+\dot{\tilde{q}}_{p}(k)\right)
\end{aligned}
$$

where

$$
A_{a}=\left[\begin{array}{cccc}
0 & I & 0 & 0 \\
-K_{m} & 0 & 0 & 0 \\
0 & 0 & 0 & I \\
0 & 0 & -K_{m} & -L
\end{array}\right], \quad B_{c}=\left[\begin{array}{c}
0 \\
I \\
0 \\
-I
\end{array}\right]
$$

We also assume that sampling rate is small so that the exponential matrix can be approximated by the first-order Taylor expansion $e^{A_{a} \Delta t} \approx I+A_{a} \Delta t$. After discretizing Equation (28), we obtain

$$
\begin{aligned}
& x_{a}(k+1)=A_{b} x_{a}(k)+A_{b u} x_{a}(k)+B_{b} H\left[W_{m}^{\mathrm{T}}(k) \tilde{\theta}(k)\right] \\
& \tilde{\theta}(k+1)=\tilde{\theta}(k)-\Delta t \Gamma W_{m}(k) \gamma\left(\dot{e}_{p}(k)+\dot{\tilde{q}}_{p}(k)\right)
\end{aligned}
$$

where

$$
\begin{gathered}
A_{b} \approx \exp \left(A_{\tilde{a}} \Delta t\right), \quad A_{b u}=A_{c u} \Delta t \\
B_{b} \approx \int_{0}^{\Delta t} \exp \left(A_{\tilde{a}}(\Delta t-s)\right) B_{c} \mathrm{~d} s \\
A_{\tilde{a}}=\left[\begin{array}{cccc}
0 & I & 0 & 0 \\
-K_{m} & -\gamma & 0 & -\gamma \\
0 & 0 & 0 & I \\
0 & \gamma & -K_{m} & \gamma-L
\end{array}\right]
\end{gathered}
$$


Using the discrete time version of averaging technique [7], the average dynamics of the parameter estimation error equation in Equation (29) is given by

$$
\tilde{\theta}_{\mathrm{avg}}(k+1) \approx\left(I-\Delta t \Gamma A V G\left(W_{m}(k) \hat{M}_{d}\left(W_{m}^{\mathrm{T}}(k)\right)\right) \tilde{\theta}_{\mathrm{avg}}(k)\right.
$$

where $\hat{M}_{d}$ is the transfer function matrix,

$$
\hat{M}_{d}(z)=C_{b}\left(z I-A_{b}\right)^{-1} B_{b}, \quad C_{b}=\left[\begin{array}{llll}
0 & \gamma & 0 & \gamma
\end{array}\right]
$$

and $z$ is the $Z$-transform variable. Equation (30) is the sampled and zero-order hold input version of the continuous time result obtained in Reference [5]. Therefore, $\hat{M}_{d}$ in Equation (31) is given by

$$
\hat{M}_{d}(z)=\left(1-z^{-1}\right) Z\left(L^{-1}\left(\frac{1-e^{-s \Delta t}}{s} \hat{M}_{o}(s)\right)\right)
$$

where $L^{-1}$ and $Z$, respectively, denote the inverse Laplace and $Z$-transforms, and $\hat{M}_{o}(s)$ is given in Reference [5] as $\hat{M}_{o}(s)=\operatorname{diag}\left\{\hat{M}_{o 1}(s), \hat{M}_{o 2}(s)\right\}$, where

$$
\begin{aligned}
& \hat{M}_{01}(s)=\frac{\gamma_{1} L_{1} s^{2}}{S^{4}+L_{1} s^{3}+\left(2 \omega_{1}^{2}+\gamma_{1} L_{1}\right) s^{2}+\omega_{1}^{2} L_{1} s+\omega_{1}^{4}} \\
& \hat{M}_{02}(s)=\frac{\gamma_{2} L_{2} s^{2}}{S^{4}+L_{2} s^{3}+\left(2 \omega_{2}^{2}+\gamma_{2} L_{2}\right) s^{2}+\omega_{2}^{2} L_{2} s+\omega_{2}^{4}}
\end{aligned}
$$

Since the sampling frequency $(2 \pi / \Delta t)$ is larger than the reference resonant frequencies $\omega_{1}$ and $\omega_{2}$, the filtered steady state response through $\hat{M}_{d}\left(W_{m}^{\mathrm{T}}\right)$ is given by

$$
\hat{M}_{d}\left(W_{m}^{\mathrm{T}}\right)=\left[\begin{array}{cc}
X_{0} \sin \left(\omega_{1} k\right) & 0 \\
A_{1} Y_{0} \sin \left(\omega_{2} k+\phi_{1}\right) & A_{2} X_{0} \sin \left(\omega_{1} k+\phi_{2}\right) \\
0 & Y_{0} \sin \left(\omega_{2} k\right) \\
X_{0} \omega_{1} \cos \left(\omega_{1} k\right) & 0 \\
A_{1} Y_{0} \omega_{2} \cos \left(\omega_{2} k+\phi_{1}\right) & A_{2} X_{0} \omega_{1} \cos \left(\omega_{1} k+\phi_{2}\right) \\
0 & Y_{0} \omega_{2} \cos \left(\omega_{2} k\right) \\
-2 A_{1} Y_{0} \omega_{2} \cos \left(\omega_{2} k+\phi_{1}\right) & 2 A_{2} X_{0} \omega_{1} \cos \left(\omega_{1} k+\phi_{2}\right)
\end{array}\right]^{\mathrm{T}}
$$

where

$$
\begin{gathered}
A_{1}=\frac{\gamma_{1} L_{1} \omega_{2}^{2}}{\sqrt{\left(\gamma_{1} L_{1} \omega_{2}^{2}-\left(\omega_{1}^{2}-\omega_{2}^{2}\right)^{2}\right)^{2}+L_{1}^{2} \omega_{2}^{2}\left(\omega_{1}^{2}-\omega_{2}^{2}\right)^{2}}} \\
\phi_{1}=\tan ^{-1} \frac{L_{1} \omega_{2}\left(\omega_{1}^{2}-\omega_{2}^{2}\right)}{\gamma_{1} L_{1} \omega_{2}^{2}-\left(\omega_{1}^{2}-\omega_{2}^{2}\right)^{2}}
\end{gathered}
$$




$$
\begin{gathered}
A_{2}=\frac{\gamma_{2} L_{2} \omega_{1}^{2}}{\sqrt{\left(\gamma_{2} L_{2} \omega_{1}^{2}-\left(\omega_{1}^{2}-\omega_{2}^{2}\right)^{2}\right)^{2}+L_{2}^{2} \omega_{1}^{2}\left(\omega_{1}^{2}-\omega_{2}^{2}\right)^{2}}} \\
\phi_{2}=\tan ^{-1} \frac{-L_{2} \omega_{1}\left(\omega_{1}^{2}-\omega_{2}^{2}\right)}{\gamma_{2} L_{2} \omega_{1}^{2}-\left(\omega_{1}^{2}-\omega_{2}^{2}\right)^{2}}
\end{gathered}
$$

Note that Equation (32) has the same form as its continuous time counterpart equation in Reference [5]. Therefore, every convergence property mentioned in the continuous time case is preserved here.

Applying the decoupling condition $X_{0} \omega_{1}=Y_{0} \omega_{2}$, the bandwidth of the adaptive controlled gyroscope is approximately given by

$$
\tilde{\Omega}_{z \text { avg }}(k+1) \approx\left(1-2 \Delta t \gamma_{\Omega} X_{0}^{2} \omega_{1}^{2}\right) \tilde{\Omega}_{z \text { avg }}(k)
$$

or

$$
\dot{\tilde{\Omega}}_{z \mathrm{avg}} \approx-2 \gamma_{\Omega} X_{0}^{2} \omega_{1}^{2} \tilde{\Omega}_{z \mathrm{avg}}
$$

since the parameter estimation dynamics is much slower than the sampling rate. This is exactly same result that was obtained for the continuous time observer-based adaptive control case, and the bandwidth of the adaptive controlled gyroscope is also approximately given by $B W \approx 2 \gamma_{\Omega} X_{0}^{2} \omega_{1}^{2}$. Thus, the bandwidth of a MEMS gyroscope under the observer-based discrete time adaptive control is also proportional to the adaptation gain $\gamma_{\Omega}$ and the energy of oscillation of the reference model. Other statements made in Reference [5] regarding the comparison between the analytical convergence rate of the angular rate estimate and simulation results for various resonant frequency ratios, and control and observer gains are also valid.

\subsection{Resolution analysis}

The resolution analysis for this hybrid control system that will be presented in this section is very similar to those in Reference [5]. The error expectation propagation of Equation (19) is given by

$$
E\left[x_{d}(k+1)\right]=A_{d}(k) E\left[x_{d}(k)\right]+A_{d u} E\left[x_{d}(k)\right]+C_{d}(k) \theta
$$

where $E[\cdot]$ denotes stochastic expectation. Notice that the expectation equation has the same form as its deterministic counterpart. Therefore, the mean trajectory under a stochastic environment is also biased because of the $C_{d}(k) \theta$ term. Defining covariance as $P_{d}=$ $E\left[\left(x_{d}-E\left[x_{d}\right]\right)\left(x_{d}-E\left[x_{d}\right]\right)^{\mathrm{T}}\right]$, the covariance propagation equation is given by

$$
P_{d}(k+1) \approx A_{d}(k) P_{d}(k) A_{d}^{\mathrm{T}}(k)+G_{d}(k) S_{d} G_{d}^{\mathrm{T}}(k)
$$

The covariance $P_{d}$ can easily be pre-computed independently of Equation (35). The standard deviation of the angular rate estimate error is obtained from the covariance matrix $P_{d}$ as

$$
\sigma_{\text {meas }}=\sqrt{H P_{d} H^{\mathrm{T}}}
$$

where $H=\left[0_{1 \times 14} 1\right]$. In this case, the resolution is a summation of the standard deviation of angular rate estimate error computed from (36) and the bias in the angular rate estimate. The 
ultimate achievable resolution can also be calculated by setting $S_{p}=0$ and computing $\sigma_{\text {meas }}$ with Equation (36). As in the convergence rate analysis presented in Reference [5], the same results regarding the effects of various design parameters such as control gains and parameter adaptation gains on the variance of the angular rate estimate error, can be stated in the discrete time case. In summary, the resolution of the gyroscope can be adjusted independently by the angular rate adaptation gain, without affecting the other fabrication imperfection estimation dynamics.

The effect of the quantization noise $\delta q_{d}(k)$ on the velocity estimation may be explored in a similar way as the analysis used in Section 5.1. Other noises which might be caused by the interface connection between digital processor and a MEMS gyroscope can be included in position measurement noise. Overall, gyroscope resolution can be estimated by following formula:

$$
\text { resolution }=\sqrt{\sigma_{\text {meas }}^{2}+\sigma_{\text {quan }}^{2}}
$$

where $\sigma_{\text {meas }}^{2}$ is a variance of the angular rate estimate error due to measurement noise, and $\sigma_{\text {quan }}^{2}$ is a variance of the angular rate estimate error due to quantization noise.

\section{SIMULATIONS}

A computer simulation study was conducted using the preliminary design data of the MIT-SOI MEMS gyroscope, to test the analytical results presented in this paper and verify its predicted performance. The data of some of the gyroscope parameters is given by Table I and is same as that used in Reference [5]. The numerical values for the controller used in the simulation are: $\gamma=1, \gamma_{R}=1 / 5, \gamma_{D}=1 / 10, \gamma_{\Omega}=1 / 50$, and $L=\operatorname{diag}\{1,1\}$. Notice that these numerical values are shown in non-dimensional units, which are non-dimensionalized based on the proof-mass, length of one micron and the $x$-axis nominal natural frequency.

The estimate of the angular rate response to the step input angular rate is shown in Figure 3. In this figure, the upper and lower bounds, which corresponds to the analytically estimated standard deviation calculated by Equation (37) are also plotted. Figure 4 shows the estimate of angular rate response to the sinusoidal input angular rate. These simulation results support the theoretical results obtained in the previous section, regarding predicted gyroscope bandwidth and resolution.

Table I. Key parameters of the gyroscope.

\begin{tabular}{lc}
\hline Parameter & Value \\
\hline Mass & $5.095 \times 10^{-7} \mathrm{~kg}$ \\
$x$-axis frequency & $4.17 \mathrm{kHz}$ \\
$y$-axis frequency & $5.11 \mathrm{kHz}$ \\
Quality factor & $10^{4}$ \\
Brownian noise PSD & $1.47 \times 10^{-26} \mathrm{~N}^{2} \mathrm{~s}$ \\
Position noise PSD & $1.49 \times 10^{-27} \mathrm{~m}^{2} \mathrm{~s}$ \\
\hline
\end{tabular}




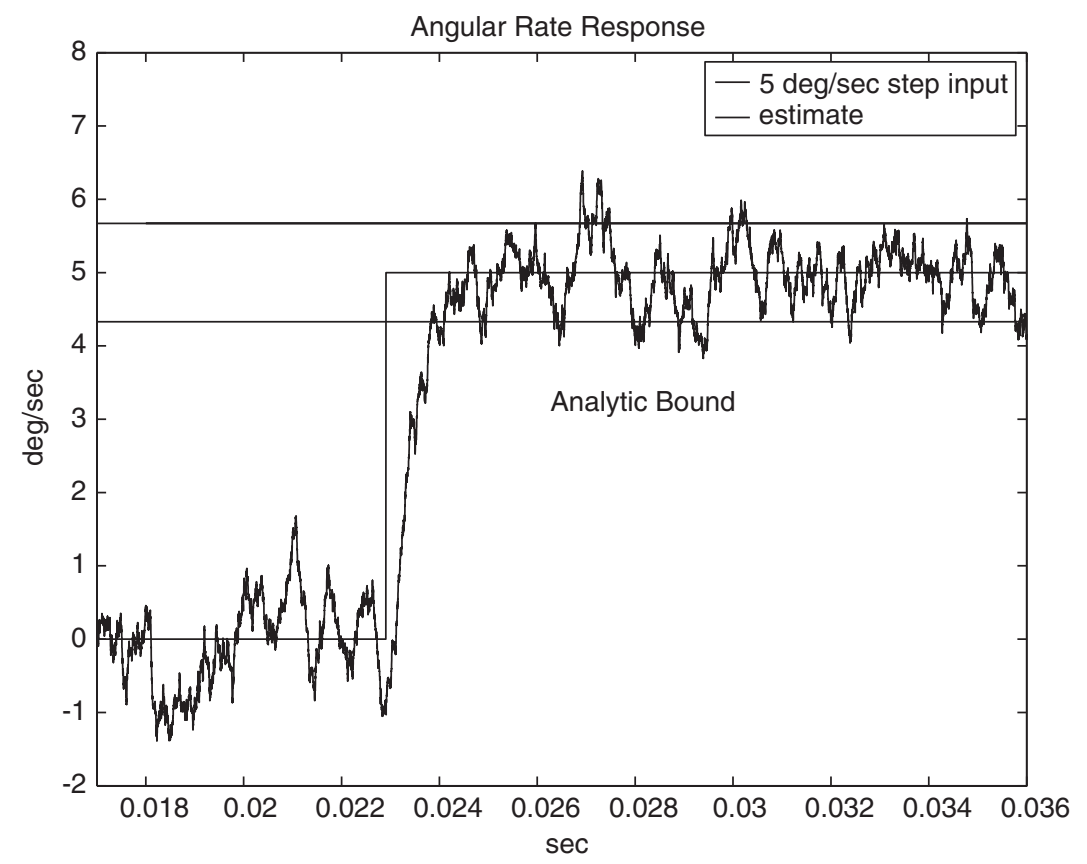

Figure 3. Time response of angular rate estimate to the $5^{\circ} / \mathrm{s}$ step input.

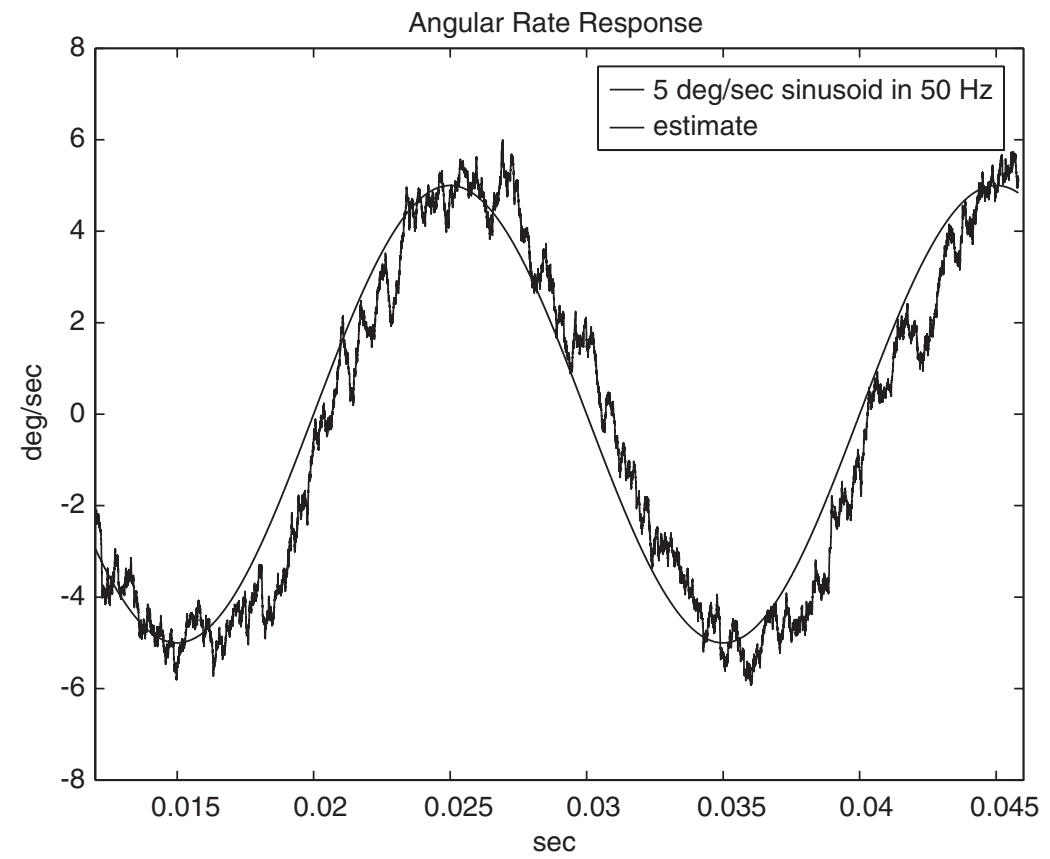

Figure 4. Time response of angular rate estimate to the $5^{\circ} / \mathrm{s}$ sinusoid input at $50 \mathrm{~Hz}$. 


\section{CONCLUSIONS}

A new adaptive control algorithm developed in Reference [5] offers several advantages such as a larger operation bandwidth, absence of zero-rate output, self-calibration and a large robustness to parameter variations. However, it is a continuous time controller. In this paper, a discrete time version of this control system was developed, which can be implemented using digital processors, and its stability was proven. The convergence and stochastic analysis presented in this paper showed that all convergence and stochastic properties of a continuous time adaptive control were preserved.

However, a stochastic analysis showed that the estimates of the angular rate and the fabrication imperfections are biased due to the signal discretization errors in the feedforward control path introduced by the sampler and holder. Thus, a two-rate discrete time control was proposed as a compromise between the measurement biases due to discretization errors and the computational burden imposed on the controller due to a fast sampling rate. In this control scheme, the parameter adaptation algorithm is updated at a slower rate than the reference signal which is used in the feedforward control. An analysis method was also developed for determining the trade-off between the controller sampling frequency and the magnitude of the angular rate estimate biased errors.

A simulation study using the preliminary design data of the MIT-SOI MEMS gyroscope was conducted, to test the analytical results derived in this paper and to verify the predicted performance of the proposed controlled schemes. Simulation results were in strong agreement with the analytically derived predicted results and performance estimates.

\section{ACKNOWLEDGEMENTS}

This research was supported by DARPA under Contract N66001-97-C-8643.

\section{REFERENCES}

1. Yazdi N, Ayazi F, Najafi K. Micromachined inertial sensors. Proceedings of IEEE 1998; 86(8):1640-1659.

2. Shkel A, Howe RT, Horowitz R. Modeling and simulation of micromachined gyroscopes in the presence of imperfection. International Conference on Modelling and Simulation of Microsystems, Puerto Rico, 1999; 605-608.

3. Jiang X, Seeger J, Kraft M, Boser BE. A monolithic surface micromachined $z$-axis gyroscope with digital output. IEEE 2000 Symposium on VLSI Circuits, Honolulu, HI, 2000; 16-19.

4. Chang S, Chia M, Castillo-Borelley P, Higdon W, Jiang Q, Johnson J, Obedier L, Putty M, Shi Q, Sparks D, Zarabadi S. An electroformed CMOS integrated angular rate sensor. Sensors and Actuators 1998; A66:138-143.

5. Park S, Horowitz R. New adaptive mode of operation for MEMS gyroscopes. ASME Journal of Dynamic Systems, Measurement and Controls 2004; 126(4):708-718.

6. Juneau TN. Micromachined dual input axis rate gyroscope. Ph.D. Thesis, U.C. Berkeley, 1997.

7. Bai EW, Fu LC, Sastry SS. Averaging analysis for discrete time and sampled data adaptive systems. IEEE Transactions on Circuits and Systems 1998; 35(2):137-148. 